Hydrol. Earth Syst. Sci., 16, 441-449, 2012

www.hydrol-earth-syst-sci.net/16/441/2012/

doi:10.5194/hess-16-441-2012

(C) Author(s) 2012. CC Attribution 3.0 License.

\title{
Transient drawdown solution for a constant pumping test in finite two-zone confined aquifers
}

\author{
C.-T. Wang ${ }^{1,2}$, H.-D. Yeh ${ }^{2}$, and C.-S. Tsai ${ }^{2}$ \\ ${ }^{1}$ Taiwan Branch, MWH Americas Inc., Taipei, Taiwan \\ ${ }^{2}$ Institute of Environmental Engineering, National Chiao Tung University, Hsinchu, Taiwan \\ Correspondence to: H.-D. Yeh (hdyeh@mail.nctu.edu.tw)
}

Received: 25 August 2011 - Published in Hydrol. Earth Syst. Sci. Discuss.: 18 October 2011

Revised: 19 January 2012 - Accepted: 23 January 2012 - Published: 13 February 2012

\begin{abstract}
The drawdown solution has been widely used to analyze pumping test data for the determination of aquifer parameters when coupled with an optimization scheme. The solution can also be used to predict the drawdown due to pumping and design the dewatering system. The drawdown solution for flow toward a finite-radius well with a skin zone in a confined aquifer of infinite extent in radial direction had been developed before. To our best knowledge, the drawdown solution in confined aquifers of finite extent with a skin zone so far has never before been presented in the groundwater literature. This article presents a mathematical model for describing the drawdown distribution due to a constantflux pumping from a finite-radius well with a skin zone in confined aquifers of finite extent. The analytical solution of the model is developed by applying the methods of Laplace transforms, Bromwich contour integral, and residue theorem. This solution can be used to investigate the effects of finite boundary and conductivity ratio on the drawdown distribution. In addition, the inverse relationship between Laplaceand time-domain variables is used to develop the large time solution which can reduce to the Thiem solution if there is no skin zone.
\end{abstract}

\section{Introduction}

The famous Theis solution (1935) was first introduced in the groundwater literature to describe the transient drawdown distribution induced by a constant pumping at a well of infinitesimal well radius in a homogeneous and isotropic confined aquifer of infinite extent. The radius of a well is in fact not zero in the real-world problems. It is well recognized that the solution developed based on the assumption of zero well radius can not give accurate drawdown predictions near the wellbore. Van Everdingen and Hurst (1949) developed the transient pressure solutions for the constant flow in finite and infinite confined reservoirs with considering the effect of well radius but neglecting the skin effect. Note that the term skin effect is used to reflect the increase or decrease of hydraulic conductivity caused by drilling practices in a region near the well. With the introduction of functions commonly occurring in groundwater flow problems, Hantush (1964) gave an analytical solution and two approximate solutions for a constant pumping in confined aquifers with a finite well radius. Chen (1984) gave a short review on the use of the remote finite boundary condition in the groundwater literature. He proposed a modified Theis equation for describing the drawdown distribution in a confined aquifer of finite extent and gave a time criterion for the use of the Theis equation to predict drawdown in a finite aquifer. Wang and Yeh (2008) gave an extensive review on the relationship between the transient solution and steady-state solution for constant-flux and constant-head tests in aquifers of finite extent and infinite extent. They mentioned that the drawdown solution of the finite aquifer, rather than the infinite aquifer, can reduce to the Thiem solution when the time becomes large enough.

A positive skin is referred to a zone near the well having lower permeability than the original formation due to well construction. On the other hand, a negative skin is a zone has higher permeability than other part of aquifer formation. With considering a finite-thickness skin or patchy zone, 
Butler (1988) and Barker and Herbert (1988) developed Laplace-domain solutions for the transient drawdown induced by a constant pumping without considering the effect of the well radius in confined aquifers. Novakowski (1989) mentioned in a study that the thickness of the skin zone may range from a few millimeters to several meters. He presented a Laplace domain drawdown solution for a confined aquifer under a constant pumping with considering the effects of skin zone and wellbore storage. Butler and Liu (1993) presented a Laplace-domain solution for drawdown due to a point-source pumping in a uniform aquifer with an arbitrarily located disk of anomalous properties. In addition, they also gave a largetime solution based on the inverse relationship between the Laplace variable and time variable. Yeh et al. (2003) presented an analytical drawdown solution for the pumping test in an infinite confined aquifer by taking into account the effects of the well storage and the finite-thickness skin. They mentioned that the effect of skin zone is negligible in short and large periods of pumping time. Perina and Lee (2006) developed a general well function in Laplace domain for constant pumping in a confined, leaky, or unconfined aquifer of infinite extent with a partially penetration well, finitethickness skin. Yet, they adopted an approach such as a finite difference method to discretize the well screen for handling non-uniform wellbore flux problems.

The existing drawdown solutions for radial two-zone confined aquifers of infinite extent under constant-flux pumping were all developed in Laplace domain except the one given by Yeh et al. (2003) which was a time domain solution. Yeh et al.'s (2003) solution is in terms of an improper integral integrating from zero to infinity and its integrand comprises a singularity at the origin. In addition, the integrand is an oscillatory function with many product terms of the Bessel functions of the first and second kinds of zero and first orders. The numerical calculation of their solution is therefore time-consuming and very difficult to achieve accurate results.

The objective of this note is to develop an analytical solution from a mathematical model similar to that of Yeh et al. (2003), except that the aquifer is of horizontally finite extent. The solution of the model is also obtained by applying the methods of Laplace transforms and Bromwich contour integral. The integration of the contour integral in Yeh et al. (2003) results in a single branch point with no singularity at zero of the complex variable. Thus, a branch cut along the negative real axis of the contour should be chosen and thus a closed contour is produced. Such a procedure finally results in a complicated solution presented in Yeh et al. (2003). On the other hand, the integration of the contour integral arisen from the Laplace domain solution in our model has a simple pole at the origin and finite number of poles at other locations. The residue theorem is therefore adapted to obtain the time domain results for the skin zone and formation zone. These two results are in terms of a logarithmic function plus a summation term, rather than an integral, with Bessel functions of the first and second kinds of orders zero and first.
This newly derived solution is much easier to calculate than that of Yeh et al. (2003) involving a singularity in the integral. In addition, a large-time solution in a simpler form is also developed by employing the relationship of small Laplace-domain variable $p$ versus large time-domain variable $t$, hereinafter referred to SPLT (Yeh and Wang, 2007), to the Laplace-domain solution. This new large-time solution is independent of time and can reduce to the Thiem equation when the skin zone is absent.

This new time-domain solution can be applied to: (1) predict the spatial and/or temporal drawdown distributions in both the skin and formation zones with known aquifer parameters such as the outer radius of the skin zone as well as the transmissivity and storage coefficient for each of the skin and aquifer zones, (2) determine the aquifer parameters if coupled with an optimization algorithm in the pumping test data analyses, (3) verify numerical codes in the prediction of the drawdown distribution in two-zone aquifer systems, and (4) perform the sensitivity analysis and assess the impacts of parameter uncertainty on the predicted drawdown.

\section{Mathematical model}

\subsection{Mathematical statement}

The assumptions involved in the development of the mathematical model are: (1) the confined aquifer is homogeneous, isotropic, and of finite extent in radial direction, (2) the well fully penetrates the aquifer and has a finite well radius, (3) a skin zone is present around the pumping well shown in Fig. 1, (4) the well discharge rate is maintained constant through out the entire pumping test.

The governing equations describing the drawdown distribution $s(r, t)$ in the skin zone and formation zone are, respectively,

$$
\begin{array}{ll}
\frac{\partial^{2} s_{1}}{\partial r^{2}}+\frac{1}{r} \frac{\partial s_{1}}{\partial r}=\frac{S_{1}}{T_{1}} \frac{\partial s_{1}}{\partial t} & r_{\mathrm{w}} \leq r<r_{1} \\
\frac{\partial^{2} s_{2}}{\partial r^{2}}+\frac{1}{r} \frac{\partial s_{2}}{\partial r}=\frac{S_{2}}{T_{2}} \frac{\partial s_{2}}{\partial t} & r_{1} \leq r<R
\end{array}
$$

where subscripts 1 and 2 denote the skin zone and formation zone, respectively, $r$ is the radial distance from the central line of the pumping well, $r_{\mathrm{w}}$ is the well radius, $r_{1}$ is the outer radius of the skin zone, $R$ is the radius of influence defined as a distance measured from the center of the well to a location where the pumping drawdown is very close to zero, $t$ is the pumping time, $S$ is the storage coefficient, and $T$ is the transmissivity.

Prior to pumping, there is no drawdown over the entire aquifer. Thus, the initial conditions for both skin zone and formation zone can be written as

$s_{1}(r, 0)=s_{2}(r, 0)=0$. 


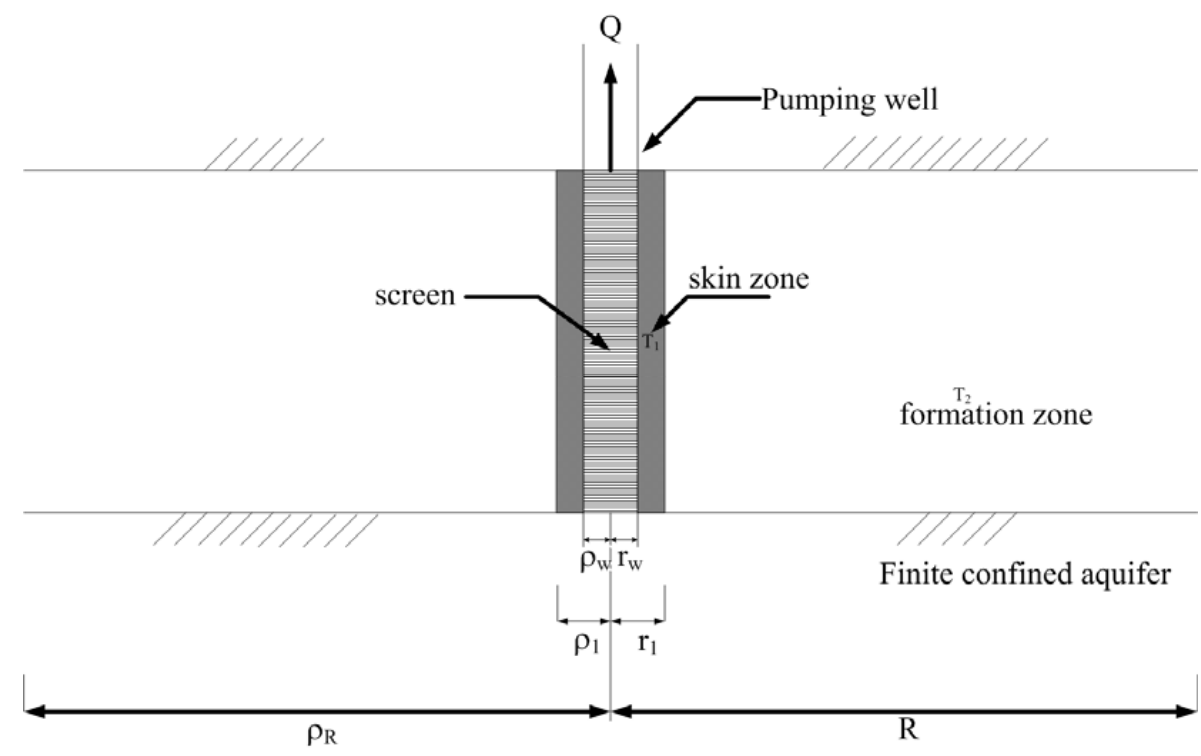

Fig. 1. Schematic diagram of the pumping test in a finite-extent confined aquifer.

In addition, the drawdown at $R$ is also zero. The flux across the well is pumped at a constant rate $Q$. Thus, the outer and inner boundary conditions can be expressed, respectively, as

$s_{2}(R, t)=0$

$\left.\frac{\mathrm{d} s_{1}}{\mathrm{~d} r}\right|_{r=r_{\mathrm{w}}}=\frac{-Q}{2 \pi r_{\mathrm{w}} T_{1}}$.

The continuity requirements for the drawdown and flux at the interface between the skin zone and formation zone are, respectively,

$s_{1}\left(r_{1}, t\right)=s_{2}\left(r_{1}, t\right)$

$T_{1} \frac{\partial s_{1}\left(r_{1}, t\right)}{\partial r}=T_{2} \frac{\partial s_{2}\left(r_{1}, t\right)}{\partial r}$.

\subsection{Laplace-domain solution}

The solutions of Eqs. (1) and (2) subject to Eqs. (3)-(7) can be easily found using the method of Laplace transforms. The results are

$\bar{s}_{1}=\frac{-Q}{4 \pi T_{2}}\left[\frac{1}{p} \frac{2 T_{2}}{r_{\mathrm{w}} T_{1} q_{1}} \frac{\Phi_{1} I_{0}\left(q_{1} r\right)-\Phi_{2} K_{0}\left(q_{1} r\right)}{\Phi_{1} I_{1}\left(q_{1} r_{\mathrm{W}}\right)+\Phi_{2} K_{1}\left(q_{1} r_{\mathrm{W}}\right)}\right]$

$\bar{s}_{2}=\frac{-Q}{4 \pi T_{2}}\left\{\frac{1}{p} \frac{2 T_{2}}{r_{\mathrm{w}} T_{1} q_{1}} \frac{\Phi_{1} I_{0}\left(q_{1} r_{1}\right)-\Phi_{2} K_{0}\left(q_{1} r_{1}\right)}{\left[\Phi_{1} I_{1}\left(q_{1} r_{\mathrm{w}}\right)+\Phi_{2} K_{1}\left(q_{1} r_{\mathrm{w}}\right)\right]} \frac{1}{\varphi}\right\}(9)$

with following lumped variables for compactness of the solutions

$\Phi_{1}=\phi \sqrt{\frac{S_{2} T_{2}}{S_{1} T_{1}}} K_{0}\left(q_{1} r_{1}\right) K_{0}\left(q_{2} r_{1}\right)-K_{1}\left(q_{1} r_{1}\right) K_{0}\left(q_{2} r_{1}\right)$

$\Phi_{2}=\phi \sqrt{\frac{S_{2} T_{2}}{S_{1} T_{1}}} I_{0}\left(q_{1} r_{1}\right) K_{0}\left(q_{2} r_{1}\right)+I_{1}\left(q_{1} r_{1}\right) K_{0}\left(q_{2} r_{1}\right)$
$\phi=\frac{I_{0}\left(q_{2} R\right) K_{1}\left(q_{2} r_{1}\right)+I_{1}\left(q_{2} r_{1}\right) K_{0}\left(q_{2} R\right)}{I_{0}\left(q_{2} R\right) K_{0}\left(q_{2} r_{1}\right)-I_{0}\left(q_{2} r_{1}\right) K_{0}\left(q_{2} R\right)}$

$\varphi=\frac{I_{0}\left(q_{2} R\right) K_{0}\left(q_{2} r\right)-I_{0}\left(q_{2} r\right) K_{0}\left(q_{2} R\right)}{I_{0}\left(q_{2} R\right) K_{0}\left(q_{2} r_{1}\right)-I_{0}\left(q_{2} r_{1}\right) K_{0}\left(q_{2} R\right)}$

where $p$ is the Laplace variable, $q_{1}=\sqrt{p S_{1} / T_{1}}, q_{2}=$ $\sqrt{p S_{2} / T_{2}}, I_{0}$ and $K_{0}$ are the modified Bessel functions of the first and second kinds of order zero, respectively, and $I_{1}$ and $K_{1}$ are the modified Bessel functions of the first and second kinds of order first, respectively.

The variables $\phi$ and $\varphi$ can reduce to $K_{1}\left(q_{2} r_{1}\right) / K_{0}\left(q_{2} r_{1}\right)$ and $K_{0}\left(q_{2} r\right) / K_{0}\left(q_{2} r_{1}\right)$, respectively, when $R$ approaches infinity. Equations (8) and (9) are then equivalent to the solutions presented in Yeh et al. (2003, p.750) as

$\bar{s}_{1}=\frac{Q}{4 \pi T_{2}}\left[\frac{1}{p} \frac{2 T_{2}}{r_{\mathrm{w}} T_{1} q_{1}} \frac{\psi_{2} K_{0}\left(q_{1} r\right)+\psi_{1} I_{0}\left(q_{1} r\right)}{\psi_{2} K_{1}\left(q_{1} r_{\mathrm{w}}\right)-\psi_{1} I_{1}\left(q_{1} r_{\mathrm{w}}\right)}\right]$

$\bar{s}_{2}=\frac{Q}{4 \pi T_{2}}\left[\frac{1}{p} \frac{2 T_{2}}{r_{\mathrm{W}} T_{1} q_{1}} \frac{\left(\psi_{2} K_{0}\left(q_{1} r_{1}\right)+\psi_{1} I_{0}\left(q_{1} r_{1}\right)\right)}{\left(\psi_{2} K_{1}\left(q_{1} r_{\mathrm{w}}\right)-\psi_{1} I_{1}\left(q_{1} r_{\mathrm{w}}\right)\right)} \frac{K_{0}\left(q_{2} r\right)}{K_{0}\left(q_{2} r_{1}\right)}\right]$

with following lumped variables

$$
\begin{aligned}
& \psi_{1}=K_{1}\left(q_{1} r_{1}\right) K_{0}\left(q_{2} r_{1}\right)-\sqrt{\frac{S_{2} T_{2}}{S_{1} T_{1}}} K_{0}\left(q_{1} r_{1}\right) K_{1}\left(q_{2} r_{1}\right) \\
& \psi_{2}=I_{1}\left(q_{1} r_{1}\right) K_{0}\left(q_{2} r_{1}\right)+\sqrt{\frac{S_{2} T_{2}}{S_{1} T_{1}}} I_{0}\left(q_{1} r_{1}\right) K_{1}\left(q_{2} r_{1}\right) .
\end{aligned}
$$

\subsection{Time-domain solution}

The transient drawdown solution in time domain can be obtained by applying the Bromwich contour integral (Carslaw and Jaeger, 1959, p.332) to the Laplace domain solution. Detailed development is shown in Appendix A and the results 
for the drawdown solutions in skin zone and formation zone are, respectively,

$$
\begin{aligned}
& s_{1}=\frac{Q}{2 \pi T_{1}}\left\{\ln \frac{r_{1}}{r}+\frac{T_{1}}{T_{2}} \ln \frac{R}{r_{1}}-\frac{\pi}{r_{\mathrm{W}}} \sum_{n=1}^{\infty} \exp \left(-\frac{T_{1}}{S_{1}} \alpha_{n}^{2} t\right)\right. \\
& \left.\times \frac{\alpha_{n}\left(J_{1}\left(\alpha_{n} r_{\mathrm{w}}\right) Y_{0}\left(\alpha_{n} r\right)-Y_{1}\left(\alpha_{n} r_{\mathrm{w}}\right) J_{0}\left(\alpha_{n} r\right)\right)}{\zeta_{n}^{2}\left[B_{n}^{2}+\left(\zeta\left(B_{n} C_{n}+A_{n} D_{n}\right)+\zeta A_{n} B_{n} / \alpha_{n}\right) / r_{1}+\zeta^{2} A_{n}\right]-\alpha_{n}^{2}}\right\}
\end{aligned}
$$

and

$s_{2}=\frac{Q}{2 \pi T_{2}}\left\{\ln \frac{R}{r}-\frac{\pi}{r_{\mathrm{w}}} \sum_{n=1}^{\infty} \exp \left(-\frac{T_{1}}{S_{1}} \alpha_{n}^{2} t\right)\right.$

$\left.\frac{\alpha_{n}\left(J_{1}\left(\alpha_{n} r_{\mathrm{w}}\right) Y_{0}\left(\alpha_{n} r_{1}\right)-Y_{1}\left(\alpha_{n} r_{1}\right) J_{0}\left(\alpha_{n} r_{\mathrm{w}}\right)\right) \times\left(Y_{0}\left(\xi \alpha_{n} R\right) J_{0}\left(\xi \alpha_{n} r\right)-Y_{0}\left(\xi \alpha_{n} r\right) J_{0}\left(\xi \alpha_{n} R\right)\right)}{\left[\varsigma_{n}^{2} B_{n}\left[B_{n}^{2}+\zeta\left(B_{n} C_{n}+A_{n} D_{n}\right) / r_{1}+\zeta A_{n} B_{n} / \alpha_{n}+\zeta^{2} A_{n}^{2}\right]-\alpha_{n}^{2}\right]}\right\}$

with following lumped variables

$$
\begin{aligned}
\varsigma_{n}= & \frac{-\alpha_{n} J_{1}\left(\alpha_{n} r_{\mathrm{w}}\right)}{-\zeta A_{n} J_{0}\left(\alpha_{n} r\right)-B_{n} J_{1}\left(\alpha_{n} r\right)} \\
A_{n}= & {\left[J_{1}\left(\xi \alpha_{n} r_{1}\right) Y_{0}\left(\xi \alpha_{n} R\right)-J_{0}\left(\xi \alpha_{n} R\right) Y_{1}\left(\xi \alpha_{n} r_{1}\right)\right] } \\
B_{n}= & J_{0}\left(\xi \alpha_{n} R\right) Y_{0}\left(\xi \alpha_{n} r_{1}\right)-J_{0}\left(\xi \alpha_{n} r_{1}\right) Y_{0}\left(\xi \alpha_{n} R\right) \\
C_{n}= & -\xi R\left[J_{1}\left(\xi \alpha_{n} r_{1}\right) Y_{1}\left(\xi \alpha_{n} R\right)-J_{1}\left(\xi \alpha_{n} R\right)\right. \\
& \left.Y_{1}\left(\xi \alpha_{n} r_{1}\right)\right]-\xi r_{1} B_{n}-\frac{A_{n}}{\alpha_{n}} \\
D_{n}= & \xi R\left[J_{1}\left(\xi \alpha_{n} R\right) Y_{0}\left(\xi \alpha_{n} r_{1}\right)-J_{0}\left(\xi \alpha_{n} r_{1}\right)\right. \\
& \left.Y_{1}\left(\xi \alpha_{n} R\right)\right]-\xi r_{1} A_{n}
\end{aligned}
$$

where $J_{0}$ and $Y_{0}$ are the Bessel functions of the first and second kinds of order zero, respectively, $J_{1}$ and $Y_{1}$ are the Bessel functions of the first and second kinds of order first, respectively, $\xi=\sqrt{T_{1} S_{2} / T_{2} S_{1}}, \zeta=\sqrt{S_{2} T_{2} / S_{1} T_{1}}$, and $\pm \alpha_{n}$ are the roots of

$\left[\alpha J_{1}\left(\xi \alpha r_{1}\right) Y_{0}(\xi \alpha R)-\alpha J_{0}(\xi \alpha R) Y_{1}\left(\xi \alpha r_{1}\right)\right]$

$$
\begin{aligned}
& \times \zeta\left[Y_{1}\left(\alpha r_{\mathrm{w}}\right) J_{0}\left(\alpha r_{1}\right)-Y_{0}\left(\alpha r_{1}\right) J_{1}\left(\alpha r_{\mathrm{w}}\right)\right] \\
& +\left[J_{0}\left(\xi \alpha r_{1}\right) Y_{0}(\xi \alpha R)-J_{0}(\xi \alpha R) Y_{0}\left(\xi \alpha r_{1}\right)\right] \\
& \times\left[\alpha Y_{1}\left(\alpha r_{1}\right) J_{1}\left(\alpha r_{\mathrm{w}}\right)-\alpha J_{1}\left(\alpha r_{1}\right) Y_{1}\left(\alpha r_{\mathrm{w}}\right)\right]=0 .
\end{aligned}
$$

When the skin zone is absence, Eq. (19) can reduce to

$$
\begin{aligned}
s & =\frac{Q}{2 \pi T}\left\{\ln \frac{R}{r}-\frac{\pi}{r_{\mathrm{w}}} \sum_{n=1}^{\infty} \exp \left(-\frac{T}{S} \alpha_{n}^{2} t\right)\right. \\
& \left.\times \frac{\left(J_{1}\left(\alpha_{n} r_{\mathrm{W}}\right) Y_{0}\left(\alpha_{n} r\right)-Y_{1}\left(\alpha_{n} r_{\mathrm{W}}\right) J_{0}\left(\alpha_{n} r\right)\right)}{\alpha_{n}\left[J_{1}^{2}\left(\alpha_{n} r_{\mathrm{w}}\right)-J_{0}^{2}\left(\alpha_{n} R\right)\right] / J_{0}^{2}\left(\alpha_{n} R\right)}\right\}
\end{aligned}
$$

where $\alpha_{n}$ become the root of $J_{1}\left(\alpha r_{\mathrm{w}}\right) Y_{0}(\alpha R)-Y_{1}\left(\alpha r_{\mathrm{w}}\right)$ $J_{0}(\alpha R)=0$. Note that Eq. (26) is exactly the same as the equation presented in Wang and Yeh (2008, Eq. 11). Additionally, the steady-state solution can be obtained from Eqs. (18) and (19) when time approaches infinity.

\subsection{Large-time solution}

The drawdown solution for two-zone confined aquifers of finite-extent at large times can be obtained by applying the SPLT technique and L'Hospital rule to Eqs. (8) and (9). Some limits of the Bessel functions with small arguments are given as $I_{0}(x) \sim 1, I_{1}(x) \sim x / 2, K_{0}(x) \sim-\ln (x)$, and $K_{1}(x) \sim 1 / x$ when $x$ approaches zero (Abramowitz and Stegun, 1979, p.375). For small $p$, the Laplace-domain drawdown solutions for skin zone and formation zone can then be obtained, respectively, as

$\bar{s}_{1}(r, p)=\frac{Q}{2 \pi T_{1} p}\left(\ln \frac{r_{1}}{r}+\frac{T_{1}}{T_{2}} \ln \frac{R}{r_{1}}\right)$

$\bar{s}_{2}(r, p)=\frac{Q}{2 \pi T_{2} p} \ln \frac{R}{r}$.

Furthermore, the drawdown solutions at large-times can then be obtained by taking the inverse Laplace transform to Eqs. (27) and (28) as

$s_{1}(r, t)=\frac{Q}{2 \pi T_{1}}\left(\ln \frac{r_{1}}{r}+\frac{T_{1}}{T_{2}} \ln \frac{R}{r_{1}}\right)$

$s_{2}(r, t)=\frac{Q}{2 \pi T_{2}} \ln \frac{R}{r}$.

In fact, Eqs. (29) and (30) can also be obtained by applying the Tauberian theorm (Sneddon, 1972) to Eqs. (8) and (9). This result indicates that the drawdown solution can reach steady state in confined aquifers of finite extent as declared in Wang and Yeh (2008). In addition, both Eqs. (29) and (30) can reduce to the Thiem solution if there is no skin zone, i.e. $r_{1}=r_{\mathrm{w}}$ and $T_{1}=T_{2}$.

\subsection{Dimensionless solution}

Dimensionless variables are introduced as follows: $\kappa=$ $T_{2} / T_{1}, \gamma=S_{2} / S_{1}, \tau=T_{2} t / S_{2} r_{\mathrm{w}}^{2}, \rho=r / r_{\mathrm{w}}, \rho_{1}=r_{1} / r_{\mathrm{w}}, \rho_{R}=$ $R / r_{\mathrm{w}}$, and $s_{D}=s\left(4 \pi T_{2}\right) /\left(4 \pi T_{2}\right) / Q$ where $\kappa$ represents conductivity ratio, $\gamma$ represents the ratio of storage coefficient, $\rho$ represents dimensionless distance, $\rho_{1}$ represents dimensionless skin thickness, $\rho_{R}$ represents dimensionless distance of the outer boundary, and $s_{D}$ represents the transient distribution of dimensionless drawdown. The drawdown solutions in Eqs. (18) and (19) then becomes

$$
\begin{aligned}
& s_{1 D}=2 \kappa\left\{\ln \frac{\rho_{1}}{\rho}+\frac{1}{\kappa} \ln \frac{\rho_{R}}{\rho_{1}}-\pi \sum_{n=1}^{\infty} \exp \left(-\frac{\gamma}{\kappa} \beta_{n}^{2} \tau\right)\right. \\
& \left.\times \frac{\beta_{n}\left(J_{1}\left(\beta_{n}\right) Y_{0}\left(\beta_{n} \rho\right)-Y_{1}\left(\beta_{n}\right) J_{0}\left(\beta_{n} \rho\right)\right)}{\varsigma_{D n}^{2}\left[b_{n}^{2}+\left(\zeta\left(b_{n} c_{n}+a_{n} d_{n}\right)+\zeta a_{n} b_{n} / \beta_{n}\right) / \rho_{1}+\zeta^{2} a_{n}^{2}\right]-\beta_{n}^{2}}\right\} \\
& s_{2 D}=2 \kappa\left\{\ln \frac{\rho_{R}}{\rho}-\pi \sum_{n=1}^{\infty} \exp \left(-\frac{\gamma}{\kappa} \beta_{n}^{2} \tau\right)\right. \\
& \frac{\beta_{n}\left(J_{1}\left(\beta_{n}\right) Y_{0}\left(\beta_{n} \rho_{1}\right)-Y_{1}\left(\beta_{n} \rho_{1}\right) J_{0}\left(\beta_{n}\right)\right) \times\left(Y_{0}\left(\xi \beta_{n} \rho_{R}\right) J_{0}\left(\xi \beta_{n} \rho\right)-Y_{0}\left(\xi \beta_{n} \rho\right) J_{0}\left(\xi \beta_{n} \rho_{R}\right)\right)}{\left[\zeta ^ { 2 } D _ { n } \left[b_{n}+\left(\zeta \left(b_{n} c_{n}+\alpha_{n}\right.\right.\right.\right.}
\end{aligned}
$$


where $\beta_{n}=r_{\mathrm{w}} \alpha_{n}$ are the roots of

$$
\begin{aligned}
& {\left[\beta J_{1}\left(\xi \beta \rho_{1}\right) Y_{0}\left(\xi \beta \rho_{R}\right)-\beta J_{0}\left(\xi \beta \rho_{R}\right) Y_{1}\left(\xi \beta \rho_{1}\right)\right]} \\
& \quad \times \zeta\left[Y_{1}(\beta) J_{0}\left(\beta \rho_{1}\right)-Y_{0}\left(\beta \rho_{1}\right) J_{1}(\beta)\right] \\
& \quad+\left[J_{0}\left(\xi \beta \rho_{1}\right) Y_{0}\left(\xi \beta \rho_{R}\right)-J_{0}\left(\xi \beta \rho_{R}\right) Y_{0}\left(\xi \beta \rho_{1}\right)\right] \\
& \quad \times\left[\beta Y_{1}\left(\beta \rho_{1}\right) J_{1}(\beta)-\beta J_{1}\left(\beta \rho_{1}\right) Y_{1}(\beta)\right]=0
\end{aligned}
$$

and

$$
\begin{aligned}
\varsigma_{D n}= & \frac{-\beta_{n} J_{1}\left(\beta_{n}\right)}{-\zeta a_{n} J_{0}\left(\beta_{n} \rho_{1}\right)-b_{n} J_{1}\left(\beta_{n} \rho_{1}\right)} \\
a_{n}= & J_{1}\left(\xi \beta_{n} \rho_{1}\right) Y_{0}\left(\xi \beta_{n} \rho_{R}\right)-J_{0}\left(\xi \beta_{n} \rho_{R}\right) Y_{1}\left(\xi \beta_{n} \rho_{1}\right) \\
b_{n}= & J_{0}\left(\xi \beta_{n} \rho_{R}\right) Y_{0}\left(\xi \beta_{n} \rho_{1}\right)-J_{0}\left(\xi \beta_{n} \rho_{1}\right) Y_{0}\left(\xi \beta_{n} \rho_{R}\right) \\
c_{n}= & -\xi \rho_{R}\left[J_{1}\left(\xi \beta_{n} \rho_{1}\right) Y_{1}\left(\xi \beta_{n} \rho_{R}\right)-J_{1}\left(\xi \beta_{n} \rho_{R}\right)\right. \\
& \left.Y_{1}\left(\xi \beta_{n} \rho_{1}\right)\right]-\xi \rho_{1} b_{n}-\frac{a_{n}}{\beta_{n}} \\
d_{n}= & \xi \rho_{R}\left[J_{1}\left(\xi \beta_{n} \rho_{R}\right) Y_{0}\left(\xi \beta_{n} \rho_{1}\right)-J_{0}\left(\xi \beta_{n} \rho_{1}\right)\right. \\
& \left.Y_{1}\left(\xi \beta_{n} \rho_{R}\right)\right]-\xi \rho_{1} a_{n} .
\end{aligned}
$$

The numerical calculations for Eqs. (31) and (32) are achieved by finding the roots of Eq. (33) first using Newton's method and then adding the summation term for $n$ up to 100 . Generally, the results have accuracy to the fifth decimal place.

\section{Advantages and applications of the present solution}

\subsection{Advantages over the existing solutions}

The analytical solution developed herein has the following two advantages over Yeh et al.'s (2003) solution. First, the present solutions can give the same predicted drawdowns as Yeh et al.'s (2003) solution if the outer boundary distance in the present solution is very large. In other words, the solutions presented by Yeh et al. (2003) can be considered as a special case of the present solution. Second, the transient drawdown solution given by Yeh et al. (2003) is in terms of an improper integral with the range from zero to infinity. In addition, their solution is rather difficult to accurately calculate because of the singularity occurring at the origin. In contrast, the present solution is composed of infinite series and can be easily calculated with accuracy to fifth decimal.

\subsection{Potential applications}

An aquifer system with the presence of skin zone can be characterized by five parameters, i.e. the outer radius of the skin zone and the transmissivity and storage coefficient for each of the skin and aquifer zones. If those parameters are known, the present solution can be used to predict the spatial or temporal drawdown distributions in both the skin and formation zones and explore the physical insight of the constant-flux test in two-zone aquifer systems. On the other hand, those five parameters can be determined via the data analyses if their values are not unknown. The determination of unknown parameters is in fact a subject of inverse problems. The typecurve approach is commonly used for the determination of aquifer parameters. However, it is almost impossible to develop type-curves for the parameter estimation because the unknowns of a two-zone aquifer are too many. An alternative way to determine those five unknown parameters is to use the present solution in conjunction with the algorithm of extended Kalman filter (e.g. Leng and Yeh, 2003; Yeh and Huang, 2005) or a heuristic optimization approach such as genetic algorithm or simulated annealing (e.g. Yeh et al., 2007, 2009). It is of interest to note that Yeh et al. (2009) developed a numerical approach composed of the drawdown solution developed by Yeh et al. (2003) and the algorithm of simulated annealing. This approach was used to analyze 84 hypothetical drawdown data sets which included 14 different scenarios and each scenario contained 6 cases. The analyzed results demonstrated that their approach could give reasonably good estimations to the thickness of the skin zone and four aquifer parameters at the same time.

The present solution can also be used to verify recently developed numerical codes for predicting the drawdown distribution in two-zone aquifer systems. Generally, the sensitivity analysis (Liou and Yeh, 1997) can be performed to assess the impacts of parameter uncertainty on the predicted drawdown. If the predicted drawdown is very sensitive to a specific parameter, a small change in that parameter will result in a large change in the predicted drawdown. On the contrary, the change in a less sensitive parameter has little influence on the predicted result, reflecting a fact that a less sensitive parameter is difficult to be accurately estimated. With the present solution, one can easily perform the sensitivity analysis for two-zone confined aquifer systems to assess the overall responsiveness and sensitivity to targeted parameters (e.g. Huang and Yeh, 2007).

\section{Results and discussion}

Yeh et al. (2003) had investigated the effects of the parameters including the skin type, skin thickness and well radius on the drawdown distribution for two-zone aquifer systems. This study therefore concentrates on the effects of finite boundary and conductivity ratio on the drawdown 


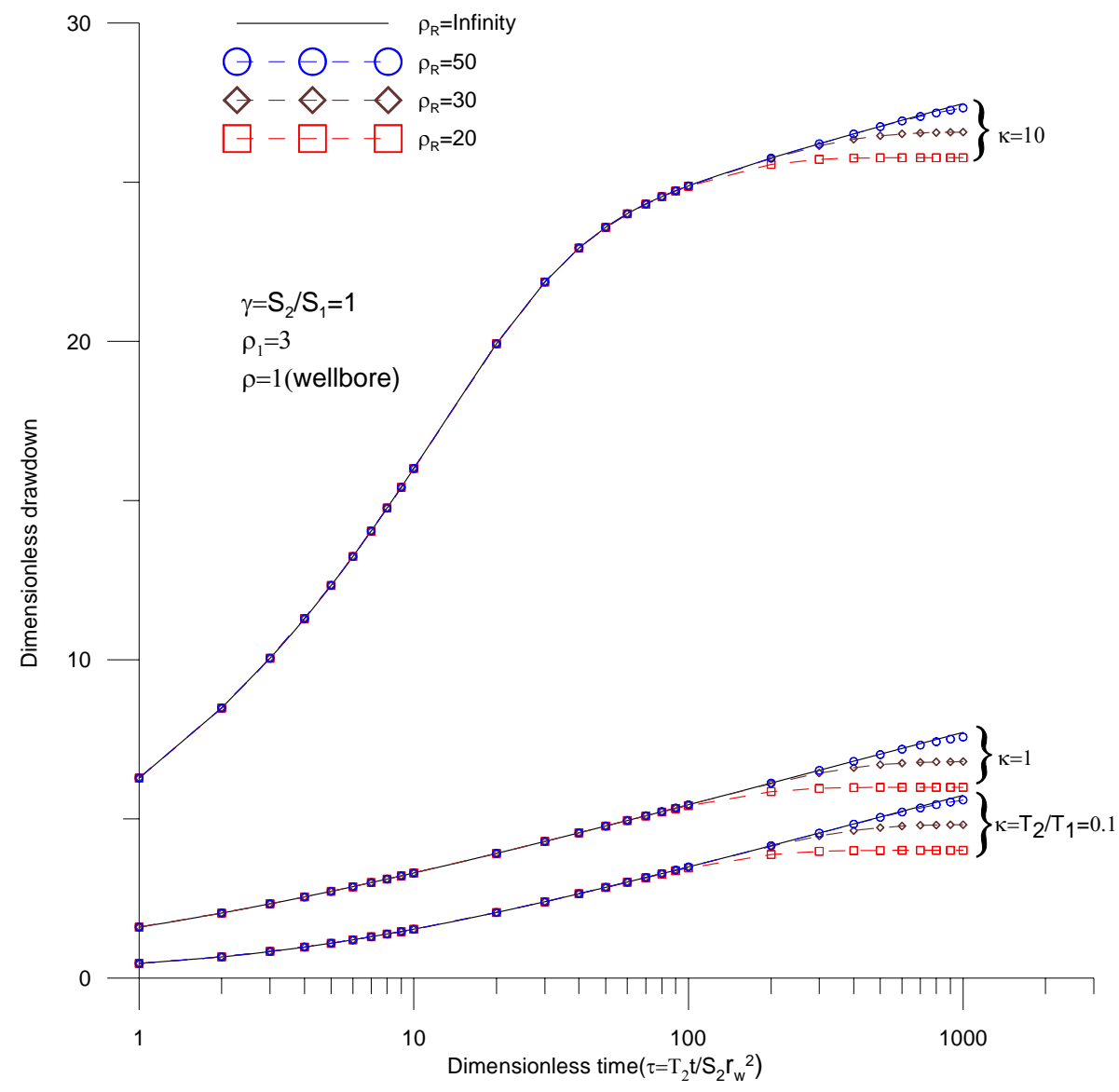

Fig. 2. The predicted drawdown curves at $\rho=1$ (wellbore) for infinite aquifers denoted by the solid line and for finite aquifers having the outer boundary distances of 20,30 , and 50 represented by the dashed lines with the symbols of circle, diamond, and square, respectively, for $\rho_{1}=3, \gamma=1$ and $\kappa=0.1,1$ and 10 .

distribution. Figures 2 and 3 depict the dimensionless predicted drawdowns at $\rho=1$ (at wellbore) and 10 (i.e. in the formation zone), respectively, when $\rho_{1}=3$ for $\kappa=0.1,1$, and 10 and $\rho_{R}=20,30$, and 50. Note that $\kappa$ less than one denotes for the case of a negative skin and greater than one for the case of a positive skin. Figure 2 shows the comparison of the wellbore drawdown in the aquifer of finite-extent to that in the one of infinite extent. Both drawdown curves match very well before $\tau<100$. However, the curves gradually deviate from one another after $\tau>100$, indicating that the solution of finite aquifers is no longer suitable to approximate the solution of infinite aquifer at large times because of the effect of the finite outer boundary on the drawdown distribution. Moreover, the drawdown solution of finite aquifers tends to be stabilized when the time becomes very large. On the other hand, the wellbore drawdown in infinite aquifers continuously increases with dimensionless time. Figure 2 also demonstrates the effect of skin property on the wellbore drawdown distribution. The aquifer with a positive skin has larger wellbore drawdowns than the one with a negative skin at the same pumping rate. Figure 3 presents the drawdown distributions at $\rho=10$ for $\rho_{1}=3$ and $\kappa=0.1,1$, and 10 . It reveals that the drawdown in an aquifer with a negative skin is larger than that in the one with a positive skin. In other words, the effect of skin property on the drawdown distribution in the formation zone is opposed to that at the wellbore. The difference in drawdown distribution between aquifers with $\kappa=1$ and 10 at $\rho=1$ shown in Fig. 2 is significantly larger than those with $\kappa=0.1$ and 1 at $\rho=1$ (Fig. 2) and those with $\kappa=1$ and 10 at $\rho=10$ shown in Fig. 3, indicating that the drawdown is sensitive to contrast in transmissivity for positive skin cases.

\section{Conclusions}

A mathematical model has been developed to describe the drawdown distribution for a pumping test performed in a two-zone confined aquifer of finite extent. The Laplacedomain solution of the model is obtained by applying the method of Laplace transforms. The analytical solution in time domain is then developed by the Bromwich contour 


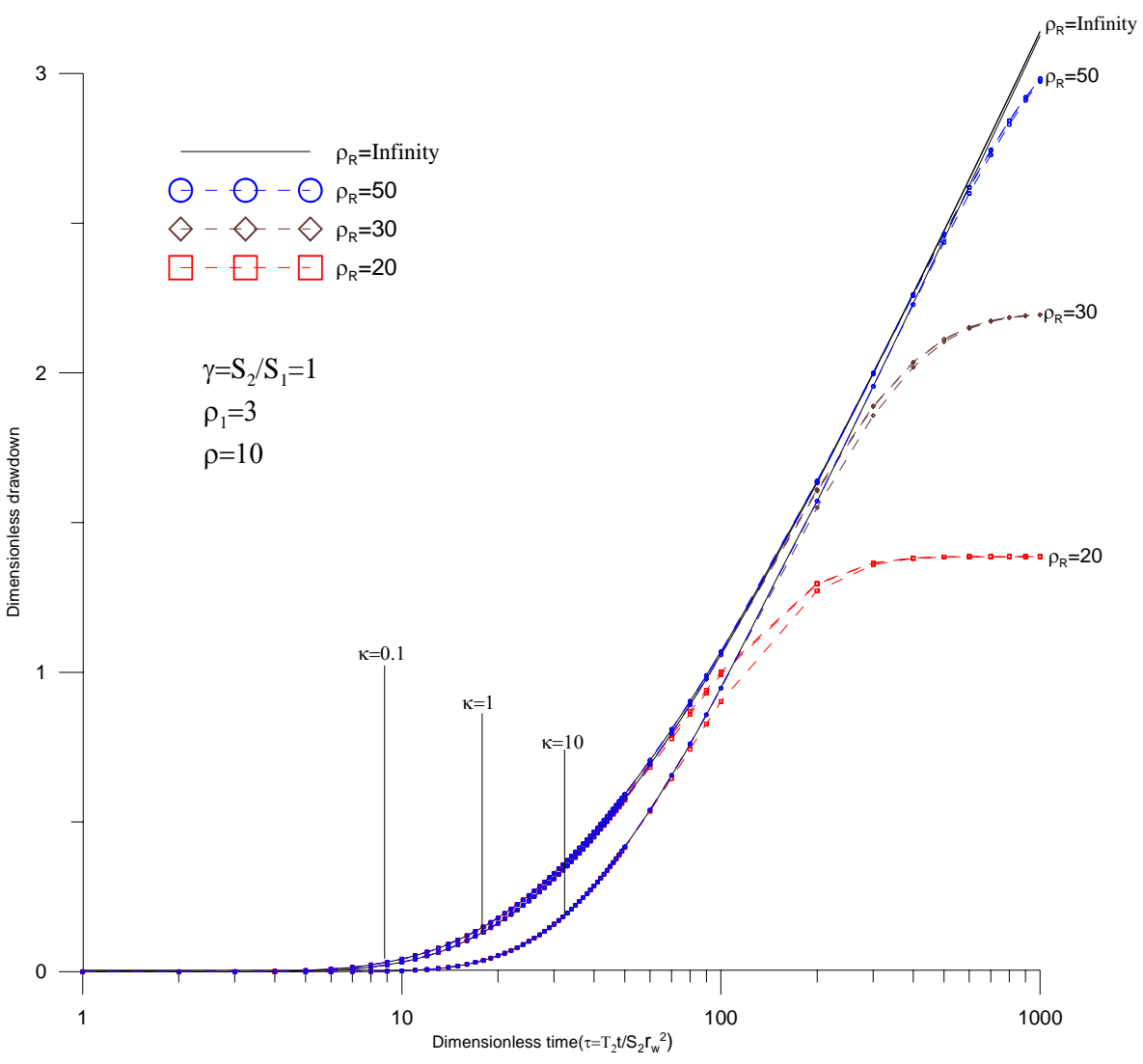

Fig. 3. The predicted drawdown curves at $\rho=10$ (in formation zone) for infinite aquifers denoted by the solid line and for finite aquifers having the outer boundary distances of 20,30 , and 50 represented by the dashed lines with the symbols of circle, diamond, and square, respectively, for $\rho_{1}=3, \gamma=1$ and $\kappa=0.1,1$ and 10 .

integral method. The drawdown distribution predicted from the analytical solution shows that the dimensionless drawdown distribution in a finite aquifer is significantly different from that in an infinite one at large pumping times. In other word, the present solution is applicable to infinite aquifers only under the condition that the time is not very large. In addition, the two-zone aquifer system is rather sensitive to the pumping in positive skin cases.

A large-time drawdown solution is also developed in this article based on the inverse relationship of Laplace- and timedomain variables. This large-time solution is exactly the same as the steady-state solution, indicating that the drawdown predicted by the present large-time solution of finite two-zone aquifers can reach steady-state at large times. In addition, the large-time solution has been shown to reduce to the Thiem solution if neglecting the presence of skin zone.

\section{Appendix A}

Derivation of Eqs. (18) and (19)

The drawdown solution in time domain, denoted as $s(t)$, obtained by applying the Bromwich integral method (Carslaw and Jaeger, 1959) to the Laplace domain solution $\bar{s}(p)$ is expressed as

$s(t)=L^{-1}\{\bar{s}(p)\}=\frac{1}{2 \pi i} \int_{r-i \infty}^{r+i \infty} e^{p t} \bar{s}(p) \mathrm{d} p$

where $i$ is an imaginary unit and $r_{e}$ is a real constant which is so large that all of the real parts of the poles are smaller than it. The graph of the Bromwich integral contains a close contour with a semicircle and a straight line parallel to the imaginary axis. According to Jordan's Lemma, the integration for the semicircle tends to be zero when it radius approaches infinity. Based on the residue theorem, Eq. (A1) can be written as

$s(t)=\sum_{n=1}^{\infty} \operatorname{Res}\left\{e^{p t} s(p) ; g_{n}\right\}$ 
where $p_{n}$ are the poles in the complex plane. There are infinite singularities in $s(p)$ and obviously one pole at $p=0$.

Introducing the following two variables

$\Delta=q_{1}\left[\Phi_{1} I_{1}\left(q_{1} r_{\mathrm{W}}\right)+\Phi_{2} K_{1}\left(q_{1} r_{\mathrm{W}}\right)\right]$

$\Psi=\left[\Phi_{1} I_{0}\left(q_{1} r\right)-\Phi_{2} K_{0}\left(q_{1} r\right)\right]$.

Equation (8) can then be expressed as

$\bar{s}_{1}=\frac{-Q}{4 \pi T_{2}} \frac{2 T_{2}}{r_{\mathrm{w}} T_{1}}\left[\frac{1}{p} \frac{\Psi(p)}{\Delta(p)}\right]$.

Let $\Delta=0$, the roots $\alpha_{n}$ in $p=p_{n}=\left(-T_{1} \alpha_{n}^{2}\right) / S_{1}$ can then be determined form Eq. (A3). Substituting $p=p_{n}=$ $\left(-T_{1} \alpha_{n}^{2}\right) / S_{1}$ into Eq. (A3) yields Eq. (25). From the following formula (Kreyszig, 1999), the residue of the pole at $p=0$ is

$\operatorname{Res}\left\{e^{p t} s(p) ; 0\right\}=\lim _{p \rightarrow 0} s(p) e^{p t}(p-0)$.

Substituting Eq. (A5) into Eq. (A6) and applying L'Hopital's rule results in

$\operatorname{Res}\left\{e^{p t} s(p) ; 0\right\}=\frac{Q}{2 \pi T_{1}}\left[\ln \frac{r_{1}}{r}+\frac{T_{1}}{T_{2}} \ln \frac{R}{r_{1}}\right]$.

The other residues at the simple pole $p=p_{n}=-T_{1} \alpha_{n}^{2} / S_{1}$ are expressed as

$\operatorname{Res}\left\{e^{p t} s(p) ; p_{n}\right\}=\lim _{p \rightarrow p_{n}} s(p) e^{p t}\left(p-p_{n}\right)$.

Applying L'Hopital's rule to Eq. (A8), the denominator term inside the brackets of Eq. (A5) becomes

$$
\begin{gathered}
{\left[p \frac{\mathrm{d} \Delta}{\mathrm{d} p}\right]_{p=-T_{1} \alpha_{n}^{2} / S_{1}}=\left[\frac{1}{2} q \frac{\mathrm{d} \Delta}{\mathrm{d} q}\right]_{q_{1}=i \alpha_{n}, q_{2}=i \kappa \alpha_{n}}} \\
=\frac{1}{2} q_{1}\left\{q_{1}\left[\Phi_{1}^{\prime} I_{1}\left(q_{1} r_{\mathrm{w}}\right)+\Phi_{2}^{\prime} K_{1}\left(q_{1} r_{\mathrm{w}}\right)\right]\right. \\
\left.+r_{\mathrm{w}} q_{1}\left[\Phi_{1} I_{0}\left(q_{1} r_{\mathrm{w}}\right)-\Phi_{2} K_{0}\left(q_{1} r_{\mathrm{w}}\right)\right]\right\}
\end{gathered}
$$

where the variables $\Phi_{1}$ and $\Phi_{2}$ are defined in Eqs. (10) and (11), respectively, and $\Phi_{1}^{\prime}$ and $\Phi_{2}^{\prime}$ are the first differentiations of $\Phi_{1}$ and $\Phi_{2}$, respectively.

To simplify Eq. (A8), a variable $\varsigma_{n}$ is assumed based on Eq. (A3) and $\Delta=0$ :

$$
\begin{aligned}
\varsigma_{n} & =\frac{q_{1} I_{1}\left(q_{1} r_{\mathrm{w}}\right) K_{0}\left(\xi q_{1} r_{1}\right)}{-\Phi_{2}\left[I_{0}\left(\xi q_{1} R\right) K_{0}\left(\xi q_{1} r_{1}\right)-I_{0}\left(\xi q_{1} r_{1}\right) K_{0}\left(\xi q_{1} R\right)\right]} \\
& =\frac{q_{1} K_{1}\left(q_{1} r_{\mathrm{w}}\right) K_{0}\left(\xi q_{1} r_{1}\right)}{\Phi_{1}\left[I_{0}\left(\xi q_{1} R\right) K_{0}\left(\xi q_{1} r_{1}\right)-I_{0}\left(\xi q_{1} r_{1}\right) K_{0}\left(\xi q_{1} R\right)\right]} .
\end{aligned}
$$

Two recurrence formulas (Carslaw and Jaeger, 1959, p.490) are adopted to eliminate the imaginary unit in Eq. (8) as follows:

$K_{v}\left(z e^{ \pm \frac{1}{2} \pi i}\right)= \pm \frac{1}{2} \pi i e^{ \pm \frac{1}{2} v \pi i}\left[-J_{v}(z) \pm i Y_{v}(z)\right]$ and

$I_{v}\left(z e^{ \pm \frac{1}{2} \pi i}\right)=e^{ \pm \frac{1}{2} v \pi i} J_{v}(z)$.

Substituting Eqs. (A11) and (A12) into Eq. (A10) yields

$$
\begin{aligned}
& \frac{-\alpha_{n} J_{1}\left(\alpha_{n} r_{\mathrm{w}}\right)}{-\zeta A_{n} J_{0}\left(\alpha_{n} r_{1}\right)-B_{n} J_{1}\left(\alpha_{n} r_{1}\right)} \\
& \quad=\frac{\alpha_{n} Y_{1}\left(\alpha_{n} r_{\mathrm{w}}\right)}{\zeta A_{n} Y_{0}\left(\alpha_{n} r_{1}\right)+B_{n} Y_{1}\left(\alpha_{n} r_{1}\right)}=\varsigma_{n} .
\end{aligned}
$$

The result of substituting Eq. (A13) into Eq. (A9) is

$$
\begin{gathered}
{\left[p \frac{\mathrm{d} \Delta}{\mathrm{d} p}\right]_{p=-T_{1} \alpha_{n}^{2} / S_{1}}=\left[\frac{1}{2} q \frac{\mathrm{d} \Delta}{\mathrm{d} q}\right]_{q_{1}=i \alpha_{n}, q_{2}=i \kappa \alpha_{n}}} \\
=\frac{1}{2 \varsigma_{n}}\left\{\varsigma _ { n } ^ { 2 } \left[B_{n}^{2}+\left(\zeta\left(B_{n} C_{n}+A_{n} D_{n}\right)\right.\right.\right. \\
\left.\left.\left.+\zeta A_{n} B_{n} / \alpha_{n}\right) / r_{1}+\zeta^{2} A_{n}^{2}\right]-\alpha_{n}^{2}\right\}
\end{gathered}
$$

where the constants appeared on the right-hand side of Eq. (A14) are defined in Eqs. (20)-(24). Similarly, the numerator of Eq. (A5) can also be obtained as

$\Psi=\frac{\pi \alpha_{n}}{2 \zeta_{n}}\left[J_{1}\left(\alpha_{n} r_{\mathrm{w}}\right) Y_{0}\left(\alpha_{n} r\right)-Y_{1}\left(\alpha_{n} r_{\mathrm{w}}\right) J_{0}\left(\alpha_{n} r\right)\right]$.

The residues at the poles $p=p_{n}=-T_{1} \alpha_{n}^{2} / S_{1}$ are

$$
\begin{aligned}
& \operatorname{Res}\left\{e^{p t} s(p) ; p_{n}\right\}=\frac{-Q}{2 \pi r_{\mathrm{w}} T_{1}} \sum_{n=1}^{\infty} \exp \left(\frac{-\alpha_{n}^{2} t T_{1}}{S_{1}}\right) \\
& \frac{\alpha_{n}\left(J_{1}\left(\alpha_{n} r_{\mathrm{w}}\right) Y_{0}\left(\alpha_{n} r\right)-Y_{1}\left(\alpha_{n} r_{\mathrm{w}}\right) J_{0}\left(\alpha_{n} r\right)\right)}{\xi_{n}^{2}\left[\left(\zeta A_{n}\right)^{2}+B_{n}^{2}+\zeta\left(\left(B_{n} C_{n}+A_{n} D_{n}\right)+A_{n} B_{n} / \alpha_{n}\right) / r_{1}\right]-\alpha_{n}} .
\end{aligned}
$$

Therefore, Eq. (A2) can be expressed as

$h(t)=\left(\operatorname{Res}\left\{e^{p t} s(p) ; 0\right\}+\operatorname{Res}\left\{e^{p t} s(p) ; p_{n}\right\}\right)$.

Finally, the solution for the drawdown distribution in the skin zone can be obtained as Eq. (18). The solution for the drawdown distribution in the formation zone can also be obtained in a similar way as Eq. (19).

Acknowledgements. This research is partially supported by the Taiwan National Science Council under the contract numbers NSC 99-2221-E-009-062-MY3, NSC 100-2221-E-009-106, and NSC 101-3113-E-007-008. The authors would like to thank the editor and two anonymous reviewers for their valuable and constructive comments that help improve the clarity of our presentation.

Edited by: A. Guadagnini 


\section{References}

Abramowitz, M. and Stegun, I. A.: Handbook of Mathematical Functions, Dover publications, New York, 1970.

Baker, J. A. and Herbert, R.: Pumping test in patchy aquifer, Ground Water, 20, 150-155, 1988.

Barry, D. A., Parlange, J. Y., and Li, L.: Approximation for the exponential integral (Theis well function), J. Hydrol., 227, 287291, 2000.

Batu, V.: Aquifer Hydraulics, John Wiley \& Sons, New York, 1998.

Butler Jr., J. J.: Pumping tests in nonuniform aquifers - The radially symmetric case, J. Hydrol., 101, 15-30, 1988.

Butler Jr., J. J. and Liu, W. Z.: Pumping tests in nonuniform aquifers: The radially asymmetric case, Water Resour. Res., 29, 259-269, 1993.

Carslaw, H. S. and Jaeger, J. C.: Conduction of Heat in Solids, 2nd Edn., Oxford University Press, London, 1959.

Chen, C. S.: A reinvestigation of the analytical solution for drawdown distributions in a finite confined aquifer, Water Resour. Res., 20, 1466-1468, doi:10.1029/WR020i010p01466, 1984.

Hantush, M. S.: Hydraulics of wells, in: Advances in Hydroscience, Academic Press, New York, 1964.

Huang, Y. C. and Yeh, H. D.: The use of sensitivity analysis in on-line aquifer parameter estimation, J. Hydrol., 335, 406-418, 2007.

Ingersoll, L. R., Adler, F. T. W., Plass, H. J., and Ingersoll, A. G.: Theory of earth heat exchangers for the heat pump, HPAC Engineering, 113-122, 1950.

Ingersoll, L. R., Zobel, O. J., and Ingersoll, A. C.: Heat conduction with engineering, geological, and other applications, 2nd Edn., University Wisconsin Press, Madison, 1954.

Kreyszig, E.: Advanced engineering mathematics, John Wiley \& Sons Inc., New York, 1999.

Leng, C. H. and Yeh, H. D.: Aquifer parameter identification using the extended Kalman filter, Water Resour. Res., 39, 1062, doi:10.1029/2001WR000840, 2003.

Liou, T. S. and Yeh, H. D.: Conditional expectation for evaluation of risk groundwater flow and solute transport: one-dimensional analysis, J. Hydrol., 199, 378-402, 1997.

Novakowski, K. S.: A Composite Analytical Model for Analysis of Pumping Tests Affected by Well Bore Storage and Finite Thickness Skin, Water Resour. Res., 25, 1937-1946, 1989.
Perina, T. and Lee, T. C.: General well function for pumping from a confined, leaky, or unconfined aquifer, J. Hydrol., 317, 239-260, 2006.

Prodanoff, J. H. A., Mansur, W. J., and Mascarenhas, F. C. B.: Numerical evaluation of Theis and Hantush-Jacob well functions, J. Hydrol., 318, 173-183, 2006.

Sneddon, I. N.: The Use of Integral Transforms, McGraw-Hill, New York, 1972.

Streltsova, T. D.: Well Testing in Heterogeneous Formations, John Wiley and Sons, Inc., New York, 45-49, 1988.

Theis, C. V.: The relation between the lowering of the piezometric surface and the rate and duration of discharge of a well using ground-water storage: Trans. Am. Geophys. Union, 16, 519524, 1935.

Tseng, P. H. and Lee, T. C.: Numerical evaluation of exponential integral: Theis well function approximation, J. Hydrol., 281, 129 146, 1998.

Van Everdingen, A. F. and Hurst, W.: The application of the Laplace transformation to flow problems in reservoirs, Pet. Trans. AIME, 186, 305-324, 1949.

Wang, C. T. and Yeh, H. D.: Obtaining the steady-state drawdown solutions of constant-head and constant-flux tests, Hydrol. Process., 22, 3456-3461, 2008.

Yeh, H. D. and Huang, Y. C.: Parameter estimation for leaky aquifers using the extended Kalman filter and considering model and data measurement uncertainties, J. Hydrol., 302, 28-45, 2005.

Yeh, H. D. and Wang, C. T.: Large-time solutions for groundwater flow problems using the relationship of small $p$ versus large $t$, Water Resour. Res., 43, W06502, doi:10.1029/2006WR005472, 2007.

Yeh, H. D., Yang, S. Y., and Peng, H. Y.: A new closed-form solution for a radial two-layer drawdown equation for groundwater under constant-flux pumping in a finite-radius well, Adv. Water Resour., 26, 747-757, 2003.

Yeh, H. D., Chang, T. H., and Lin, Y. C.: Groundwater contaminant source identification by a hybrid heuristic approach, Water Resour. Res., 43, W09420, doi:10.1029/2005WR004731, 2007.

Yeh, H. D., Chang, C. H., and Chen, Y. J.: Aquifer parameter estimation for a constant-flux test performed in a radial two-zone aquifer, J. Irrig. Drain. Eng.-ASCE, 135, 693-703, doi:10.1061/(ASCE)IR.1943-4774.0000064, 2009. 